九州大学学術情報リポジトリ

Kyushu University Institutional Repository

\title{
MATHEMATICAL FORMULATION OF GENERALIZED RELATIONAL ECOSPHERE OF LOCAL TIME-SPACES
}

Kitagawa, Toshio

Honorary Professor of Kyushu University | President, International Institute for Advanced Study of Social Information Science, Fujitsu Ltd

https://doi.org/10.5109/13351

出版情報: Bulletin of informatics and cybernetics. 21 (1/2), pp.1-18, 1984-03. Research Association of Statistical Sciences バージョン：

権利関係 : 


\title{
MATHEMATICAL FORMULATION OF GENERALIZED RELATIONAL ECOSPHERE OF LOCAL TIME-SPACES*
}

By

\author{
Tosio KitagaWA**
}

\begin{abstract}
On the basis of phenomenological consideration of each individual subjective existence in its surrounding environmental situations, local space associated with each individual knowledge-information processing system and hence local time-spaces due to its awareness are discussed. Then a generalised relational ecosphere of subjective informatical existences is formulated on the basis of category with paired morphoids. The implications are explained with respect to five cosmos formations in an ecosphere of local space-temporal existences, such as $\left(1^{\circ}\right)$ lattice structure, $\left(2^{\circ}\right)$ cell space, $\left(3^{\circ}\right)$ boundary constraints in a lattice of roads, $\left(4^{\circ}\right)$ social network system, and $\left(5^{\circ}\right)$ formal neural dynamics approach.
\end{abstract}

\section{Introduction and Summary}

This paper aims basically to present a new approach to time-space concept from the standpoint of informatics to whose logical aspects the present author has been endeavouring to contribute in a series of his papers [1] [3]. In realizing this aim, the present author is heavily due to his own works on various topics closely connected with his basic ideas on logics of informatics, as will be shown below.

In Section 2 we start with a phenomenological consideration of each individual subjective existence in its surrounding environmental situations. Our emphasis is placed upon its informatical behaviours to which we shall correspond a knowledge-information processing system (KIPS) having in general nine components. The model KIPS has been discussed in a series of our recent papers [4] [7]. To each individual subjective existence $(\mathscr{P})$ there corresponds a sequence of its local time-space represention ${ }_{\alpha} \mathcal{L}(\mathscr{P})_{\omega}$ with time slot, $(\alpha, \omega)$, as we shall show in Section 3. Each local time-space representation sequence $\left\{{ }_{\alpha} \mathcal{L}(\mathscr{Q})_{\omega}\right\}$ is then considered to be an object in an ecosphere of individual subjective existences, for whose mathematical formulation we shall introduce a category with paired morphoids, which is a generalisation of category with paired morphisms being developed by the present author since 1977 in a series of his paper [8] [10] and in his talks of twenty times in Mathematical Society of Japan. It is noted that morphoid

* This paper was presented to the 8th International Congress of Mathematical Biology, Paris, September 10th-12th, 1981.

** President, International Institute for Advanced Study of Social Information Science, Fujitsu Ltd. Honorary Professor of Kyushu University. 
is a generalisation of morphism just as grouppoid is so of group. The notion of generalised relational ecospere is underlying in such a mathematical formulation, and has been emphasised in our papers [8], [9], since we are much more concerned with environmentalogical features of assemble of subjective informatical existences than any current system science approach, as we have explictly explained in our papers [11], [12]. The present author is self-conscious of an implicit influence of Buddhism views of world existences, which is deeply underlying in Japanese traditional culture, although his own backgrounds have been mathematics, statistics, and informatics, as being explained in his paper [13]. A generalisation of interpretive structural modeling analysis due to Warfield [14], [15] can be performed to our category with paired morphoids which serves to provide us with deep structural analysis of our ecosphere of individual subjective existences, as we shall show in Section 4.

The most familiar time cosmos in classical physical sciences has been provided by Newtonian dynamics in which time cosmos can be denoted by the infinite straight line corresponding to the set of all real numbers. Our views being illustrated in these three Sections suggest us a lot of possibilities for candidate time cosmoses. In Section 5 some of them are illustrated with particular reference to cell space formulation which the present author developed with his cooperater M. Yamaguchi in a series of the papers and is being summarized in his paper [16], while others are explained in the field of neural dynamics in a series of our papers $[17] \sim[22]$.

It is hoped that some of these and/or their combinations can provide realistic and adequate pictures of spae-time cosmos regarding not only human social daily lives and/or historical descriptions but also some sort of parallel processing procedures in new computer architecture.

In conclusion of this Section, the author should point out two further points. The first point is that the author has been so much indebted to body-phenomenological considerations along the line of philosophical thoughts due to Husserl [23] and MerleauPonty [24], [25] as well as to Japanese author Ichikawa [26]. The second point is concerned with the standpoints of the author on historical views of human civilization which were explained in his monograph [27]. It is noted that a certain type of timespace existences should be introduced in discussing human civilizations as one supplement to the study of history due to Toyenbee [28].

\section{Local Space Associated with Each Individual Knowledge-information Processing System}

There are two crucial methodologial frameworks in our formulation.

The one is a local space associated with the body-subject, which is being represented by the product space composed with two coordinate spaces:

(existence coordinate subspace) $\times($ feasibility coodinate subspace).

To each of these two coordinate subspaces, a system of cutting is introduced so as to have a decomposition of the axis into subintervals. A decomposition of the coordinate subspace into its subspace is due to the intelligence level of the body subject. Generally 
speaking, the higher the intelligence level becomes, the finer the subdivision in each coordinate subspace. The details have been discussed in our papers [2], [8], [9], [10].

The other is concerned with an informatical analysis of the body-subject in terms of the following nine components:

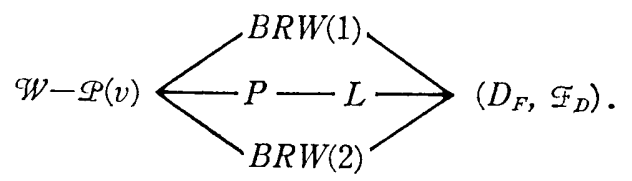

where we denote

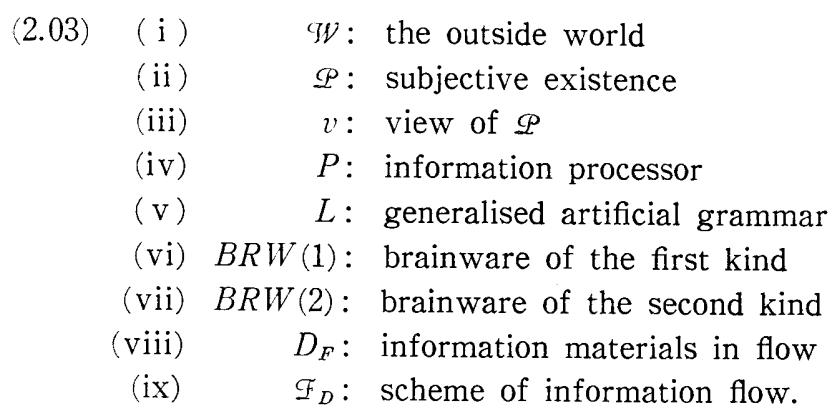

As shown in our previous papers $[4] \sim[7]$, this representation has been originated from our informational analysis of the existing data base systems. Nevertheless we emphasise an applicability of the representation to various levels of intelligence including not only $(H)$ human intelligence but also $(B)$ biological, $(M)$ mechanical, and $(S)$ social intelligences by appealing to suitable combinations of these nine components. In fact each component should be and can be interpretated so as to cover each of these varieties. For instance for certain species of biological existence for which conditional reflex theory can be adequately applied to cover most of its informatical activities, $L$ and $B R W(1)$ may be considered to be lacking and $\mathscr{T}_{D}$ is stereo-typed.

Each of these two methodological frameworks, local space and KIPS, has its own perspective rôles. Local space framework is suited to represent enthological aspects of subjective existence under its environmental circumstances. Knowledge-information processing system framework aims to take into our consideration general description of current subjective existences in the present stage of our civilization being characterized to be information-oriented society with the definite emphasis of knowledge-information features in constrast with matter-energy ones.

\section{Local Time-Space Due to Awareness}

The two fundamental frameworks explained in Section 2 will lead us to local timespace and local temporal space existence in connection with the notion of awareness which is a prototype of consciousness in human beings, as we shall explain in the following three Subsections. 


\subsection{Local time-space formations}

We shall show our local space framework will lead us to the notion of local timespace slots associated with each subjective existence.

The set of all the possible subdivisions of the existence coordinate subspace is given by $A, I, O, E$ and $U$ whose semantic implications are given by the set of correspondences:

$$
\begin{array}{ll}
A=\text { Agent }, & I=\text { Instrument } \quad O=\text { Object } \\
E=\text { Environment }, & U=\text { Umgebung (Neighbour). }
\end{array}
$$

The set of all the possible subdivisions of the feasibility subspace is given by $S b, F$, $G, O p$ and $S c$ whose semantic implications are given by the set of correspondences:

$$
\begin{aligned}
& S b=\text { Subjective }, \quad F=\text { Figuring, } \quad G=\text { Grounding } \\
& O p=\text { Operative }, \quad S c=\text { Scenary. }
\end{aligned}
$$

Here 'feasibility' is an abbreviation of subjective attitude regarding feasibility of existence which is explained in our previous papers [1], [2], [3]. In contrast with the notion of existence in Greek philosophy, one may make use of the notion of intentio in Arabic philosophy in the place of feasibility. For our detailed discussion see the papers [8], [9] and [10].

Five different types of local spaces are explained with reference to awareness of subjective existence.

EXAmple 3.1. Primitive type $1 \times 1$ This type of local space has no subdivision of either of two coordinate subspaces, existence and feasibility ones, and hence shall be represented by $1 \times 1$. An epistemological interpretation of the local space $1 \times 1$ reflects a representation of experience world where no distinction between subject and environment does exist. Hence the local space $1 \times 1$ corresponds to the world of unawareness where pure perception prevails without involving any judgement regarding existence and feasibility.

EXAMPLE 3.2. Figure-ground type $2 \times 2$ This type of local space corresponds to the subdivision of feasibility coordinate subspace into two subintervals, figuring and grounding in the sense of body phenomenology as illustrated by various philosophers. See example Ichikawa [26]. This subdivision of feasibility coordinate subspace can only be possible by having at least two different existences in the existence coordinate subspace, which implies the subdivision of the existence coordinate subspace into two parts. Hence we may and we shall introduce a matrix of the type $2 \times 2$ whose elements are denoted by $\varepsilon_{i j}(i, j=1,2)$ in the following form:

$$
\begin{array}{cc}
F & G \\
\text { Object } 1 & \left.\begin{array}{ll}
\varepsilon_{11} & \varepsilon_{12} \\
\text { Object } 2 & \\
\varepsilon_{21} & \varepsilon_{22}
\end{array}\right),
\end{array}
$$

where $F$ and $G$ denote the figurative and the grounding subspaces respectively and each $\varepsilon_{i j}$ is equal to either of one and zero with the condition that $\left(\varepsilon_{i 1}=1, \varepsilon_{i 2}=0\right)$ if the existence $i$ belongs to $F$ and $\left(\varepsilon_{i 1}=0, \varepsilon_{i 2}=1\right)$ if the existence $i$ belongs to $G$ for $i=1,2$. The motional features of these existences 1 and 2 can be illustrated by a sequence of 
matrices to the following effect.

$$
\begin{gathered}
\left(\begin{array}{ll}
0 & 1 \\
0 & 1
\end{array}\right) \\
\text { state } 1
\end{gathered} \underset{\text { state } 2}{\left(\begin{array}{ll}
1 & 0 \\
0 & 1
\end{array}\right)} \rightarrow \underset{\text { state } 3}{\left(\begin{array}{ll}
1 & 0 \\
1 & 0
\end{array}\right)} \rightarrow \underset{\text { state } 4}{\rightarrow\left(\begin{array}{ll}
1 & 0 \\
0 & 1
\end{array}\right) .}
$$

The sequence of these four states shows at the same time a change of awareness level in which the state 1 implies unawareness of subjective existence.

EXAMPLE 3.3. Basic type $3 \times 3$

$\left(1^{\circ}\right)$ A gradual increase of clearness in distinction between figuring and grounding which are basic requirement for a certain stage of perception leads to the cutting of existence coordinate subspace into three parts called $A$ (agent), $O$ (object) and $E$ (environment). In the correspondence with this subdivision of existence coordinate axis there follows the subdivision of feasibilty coordinate subspace into $S b$ (subjective), $F$ (figuring) and $G$ (grounding). The representation of the local space of this type is now given by a matrix $3 \times 3$ in such a way that

$$
\begin{array}{ccc}
\text { Sb } & F & G \\
O(i=1) & (j=2) & (j=2) \\
E(i=2)
\end{array}\left(\begin{array}{ccc}
\varepsilon_{11} & \varepsilon_{12} & \varepsilon_{12} \\
\varepsilon_{21} & \varepsilon_{22} & \varepsilon_{23} \\
\varepsilon_{31} & \varepsilon_{32} & \varepsilon_{33}
\end{array}\right),
$$

where $\varepsilon_{i j}=1$ if the $i$-th existence belongs to the $j$-th subspace of the feasibility subspace and otherwise $\varepsilon_{i j}=0$. We assume $\sum_{j=1}^{3} \varepsilon_{i j}=1(i=1,2,3)$.

$\left(2^{\circ}\right)$ The motional features in this local space can be distinguished by two cases $\left(2^{\circ}\right)_{1}$ and $\left(2^{\circ}\right)_{2}$.

$\left(2^{\circ}\right)_{1}$ Transitive case

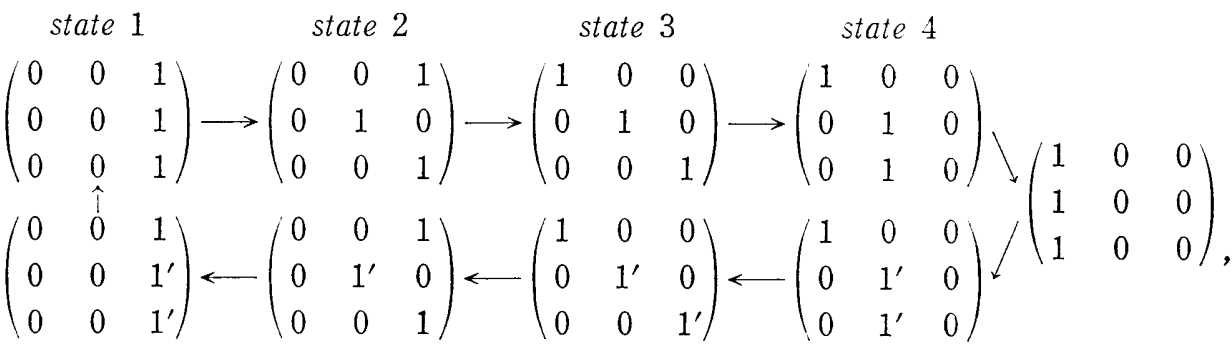

$$
\begin{aligned}
& \begin{array}{llll}
\text { state } 9 & \text { state } 8 & \text { state } 7 & \text { state } 6
\end{array}
\end{aligned}
$$

$\left(2^{\circ}\right)_{2}$ Intransitive case 


$$
\begin{aligned}
& \text { state } 1 \quad \text { state } 2 \text { state } 3 \\
& \begin{array}{l}
\left(\begin{array}{lll}
0 & 0 & 1 \\
0 & 0 & 1 \\
0 & 0 & 1
\end{array}\right) \longleftrightarrow\left(\begin{array}{lll}
0 & 0 & 1 \\
0 & 1 & 0 \\
0 & 0 & 1
\end{array}\right) \longleftrightarrow\left(\begin{array}{lll}
0 & 0 & 1 \\
0 & 1 & 0 \\
0 & 1 & 0
\end{array}\right) \\
\left(\begin{array}{lll}
0 & 0 & 1 \\
0 & 0 & 1^{\prime} \\
0 & 0 & 1^{\prime}
\end{array}\right) \longleftrightarrow\left(\begin{array}{lll}
0 & 0 & 1 \\
0 & 1^{\prime} & 0 \\
0 & 0 & 1^{\prime}
\end{array}\right) \longleftrightarrow\left(\begin{array}{lll}
0 & 0 & 1 \\
0 & 1^{\prime} & 0 \\
0 & 1^{\prime} & 0
\end{array}\right)
\end{array} \\
& \text { state } 6 \quad \text { state } 5 \text { state } 4
\end{aligned}
$$

The dashes in two cases are used in order to represent the possibility of changes of the content of existences. Each of these two sequences shows the change of levels of awareness in which the states 1 and 9 in (3.06) and the states 1 and 6 in (3.07) correspond to the state of unawareness of the subjective existence.

EXAMPLE 3.4-1. Transitive basic type $4 \times 4-(1)$

$\left(1^{\circ}\right)$ The essential aspect of local space of this type comes from extraction and clear setting up of subspace of Instrument $(I)$ in the existence coordinate space. Here the notion of Instrument is characterized by two properties:

(i) An Instrument is separated from the body subject, and is made of the existences coming from the Environment $E$.

(ii) An Instrument is operative on the Object $(O)$ under the control of body subject.

$\left(2^{\circ}\right)$ An introduction of the Instrumental subepace in the existence coordinate space induces the extraction and the clear setting up of Operative subspace in the feasibility coordinate space, and hence we have a subdivision of the feasibiiity coordinate space into four subspaces $(S b)$ Subjective, $(O p)$ Operative, $(F)$ Figuring and $(G)$ Grounding.

The representation of the local space of this type is given by a matrix $4 \times 4$ in such a way that

$$
\begin{aligned}
& \underset{(j=1)}{S b} \quad \stackrel{O p}{(j=2)} \underset{(j=3)}{F} \quad \underset{j=4}{G} \\
& \begin{array}{l}
A(i=1) \\
I(i=2) \\
O(i=3)
\end{array}\left(\begin{array}{llll}
\varepsilon_{11} & \varepsilon_{12} & \varepsilon_{13} & \varepsilon_{14} \\
\varepsilon_{21} & \varepsilon_{22} & \varepsilon_{23} & \varepsilon_{24} \\
\varepsilon_{31} & \varepsilon_{32} & \varepsilon_{33} & \varepsilon_{34} \\
\varepsilon_{41} & \varepsilon_{42} & \varepsilon_{43} & \varepsilon_{44}
\end{array}\right),
\end{aligned}
$$

where $\varepsilon_{i j}=1$ if the $i$-th existence belongs to the $j$-th subspace of the feasibility space, and otherwise $\varepsilon_{i j}=0$. We assume $\sum_{j=1}^{4} \varepsilon_{i j}=1$ for $i=1,2,3,4$.

$\left(3^{\circ}\right)$ The motional features in this local space are a refinement of transitive case $\left(2^{\circ}\right)_{1}$ in Example 3 according to its deveiopment, as can be recognized by the following illustration in which we shall now use the terminologies, unconsciousness and consciousness, because of the existence of Instrument-Operative couple. 


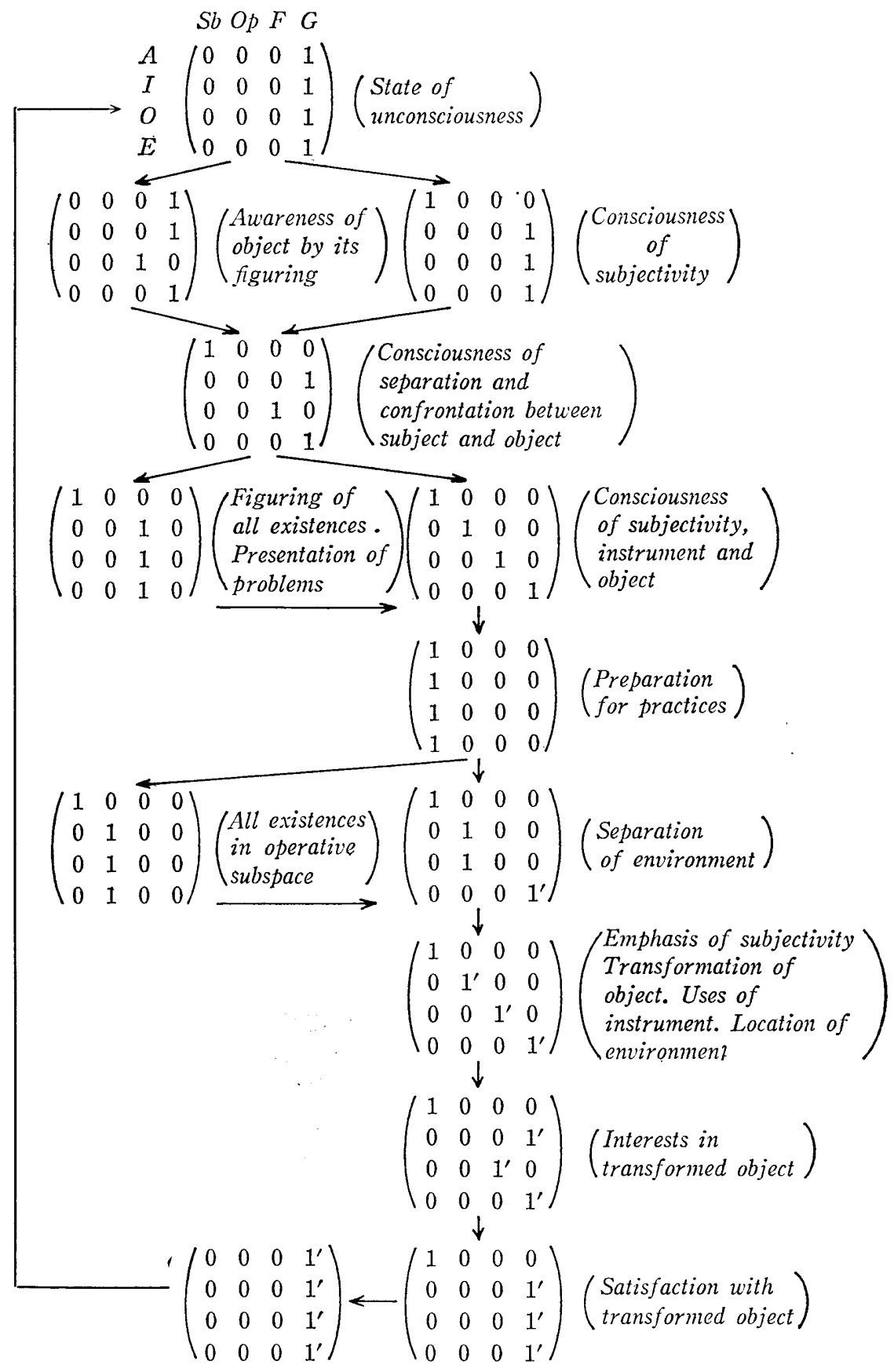

$\left(\begin{array}{l}\text { Extinction out of } \\ \text { interests }\end{array}\right)=$ (State of unconsciousness) $)$. 
EXAMPLE 3.4.-2. Intransitive basic type $4 \times 2-(2)$

$\left(1^{\circ}\right)$ The existence coordinate space in this type of local space is devided into four parts consisting of $(A)$ Agent, $(O)$ Object, $(E)$ Environment, and $(U)$ Umgebung (Neighbourhood) around the Object $(O)$.

$\left(2^{\circ}\right)$ The feasjbility coordinate space in this type of local space is divided into four parts consisting of $(S b)$ Subjective, $(F)$ Figuring, $(G)$ Grounding, and $(S c)$ Scenary subspaces.

$\left(3^{\circ}\right)$ The represention of the local space of this type is given by a matrix $4 \times 4$ in such a way that

$$
\begin{aligned}
& \underset{(j=1)}{S b} \quad \underset{(j=2)}{F} \quad \underset{(j=3)}{S c} \quad \underset{j=4}{G} \\
& \begin{array}{l}
A(i=1) \\
O(i=2) \\
U(i=3) \\
E(i=4)
\end{array}\left(\begin{array}{llll}
\varepsilon_{11} & \varepsilon_{12} & \varepsilon_{13} & \varepsilon_{14} \\
\varepsilon_{21} & \varepsilon_{22} & \varepsilon_{23} & \varepsilon_{24} \\
\varepsilon_{31} & \varepsilon_{32} & \varepsilon_{33} & \varepsilon_{34} \\
\varepsilon_{41} & \varepsilon_{42} & \varepsilon_{43} & \varepsilon_{44}
\end{array}\right),
\end{aligned}
$$

where $\varepsilon_{i j}$ is defined similarly as in Example 3.4-1.

$\left(4^{\circ}\right)$ The motional features in this local space are given by the following illustration.

$$
\begin{aligned}
& \begin{array}{llll}
S b & F & S c & G
\end{array} \\
& \begin{array}{l}
A \\
O \\
U \\
E
\end{array}\left(\begin{array}{llll}
0 & 0 & 0 & 1 \\
0 & 0 & 0 & 1 \\
0 & 0 & 0 & 1 \\
0 & 0 & 0 & 1
\end{array}\right) \rightarrow\left(\begin{array}{llll}
0 & 0 & 0 & 1 \\
0 & 1 & 0 & 0 \\
0 & 0 & 0 & 1 \\
0 & 0 & 0 & 1
\end{array}\right) \rightarrow\left(\begin{array}{llll}
0 & 0 & 0 & 1 \\
0 & 1 & 0 & 0 \\
0 & 1 & 0 & 0 \\
0 & 1 & 0 & 0
\end{array}\right) \\
& \text { state } 1 \quad \text { state } 2 \text { state } 3
\end{aligned}
$$

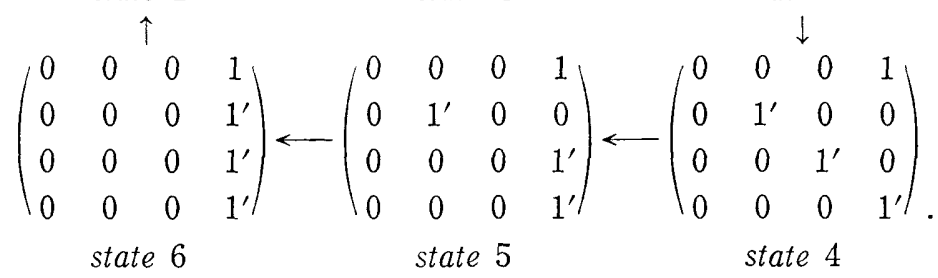

$\left(5^{\circ}\right)$ Each state in (3.11) shows the respective lovel of awareness or consciousness, where the states 1 and 6 correspond to unawareness. Here we may sometimes prefer to the notion of awareness-unawarenss in the place of consciousness-unconsciousness because of the lack of Instrument in the existence subspace and that of Operative in the feasibility subspace.

EXAMPLE 3.5. Complete basic type $5 \times 5$

$\left(1^{\circ}\right)$ The existence coordinate space in this type of local space is now divided into five subspaces consisting of $(A)$ Agent, $(I)$ Instrument, $(O)$ Object, $(U)$ Umgebung, and (E) Environment.

$\left(2^{\circ}\right)$ The feasibility coordinate space in this type of local space is now devided into five subspaces consisting of $(S b)$ Subjective, $(O p)$ Operative, $(F)$ Figuring, $(S c)$ Scenary, and $(G)$ Grounding ones. 
The representation of the local space of this type is given by a matrix $\overline{5} \times \bar{\jmath}$ in such a way that

$$
\begin{aligned}
& \underset{(j=1)}{S c} \quad \underset{(j=2)}{O p} \quad \underset{(j=3)}{F} \quad \underset{(j=4)}{S c} \quad \underset{(j=5)}{G}
\end{aligned}
$$

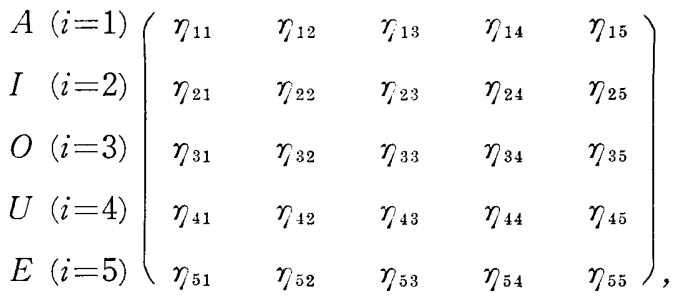

where $\eta_{i j}$ 's are defined similarly as in Examples 3.4-1 and 3.4-2.

$\left(3^{\circ}\right)$ The motional features in this local space are given similarly as in Example 3.4-1 and 3.4-2.

$\left(4^{\circ}\right)$ Because of the existence of Instrument-Operative couple we may and we shall use the paired notion consciousness-unconsciousness in this example as it was so in the case of Example 3.4-1.

Now there are two remarks. In the first place the recent developments in animal ethology are now providing us with the possibility for discussing awareness and consciousness in various animal, as we can see in Griffin [29]. Secondly it ought to be remarked that our formulation of local space is heavily due both to the thought of case grammar being originated by Filmore, who is said to be influenced by Japanese grammar, and to the motional interpretations of verbs in English, as explained for example in Ikegami [30].

\subsection{Local space-temporal existence}

The motional descriptions in the local space framework given in the previous Subsection 3.1 lead us to the notions of levels of awareness and those of consciousness, and indicate the existence of unawareness and/or unconsciousness for each individual subjective existence. In some tradional Japanese philosophy due to Do Gen (道元) based upon Buddhism, the notion of time is intrinsically connected with that of existence by maintaining that time is in existence and existence is in time. For instance refer to the explanation of Do Gen by the contemporary authors such as Takasaki and Umehara [31] pp. 279-285. According to our interpretation our local space, however, has its existence within the time slot whose beginning and ending are denoted by $\alpha$ and $\omega$ respectively. Now our local space associated with a subjective existence $\mathscr{P}$ will be denoted by ${ }_{\alpha} \mathcal{L}(\mathscr{P})_{\omega}$, or abbreviated by ${ }_{\alpha} L_{\omega}$, in which an explicit reference to $(\alpha, \omega)$ is indicated.

Now we are interested with a sequence of $\left\{\alpha_{i} \mathcal{L}(\mathscr{P}) \omega_{i} \mid i=1,2, \cdots, N\right\}$ associated with the same subjective exisrence $\mathscr{P}$. We are not in the position to be admitted to claim that $\left\{\alpha_{i}\right\}$ and $\left\{\omega_{i}\right\}$ correspond to sequences of real numbers. In stead we have to seek for an adequate mathematical description for these sequences. So for as we deal with a single subjective existence $\mathscr{P}$, we are concerned with the comparison and the connection of the nembers belonging to the sequence $\left\{\alpha_{i} \mathcal{L}(\mathscr{L}) \omega_{i} \mid i=1,2, \cdots, N\right\}$.

For the present purpose, therfore, it is sufficient for us to assume that the elements of the sets $\left\{\alpha_{i} \mid i=1,2, \cdots, N\right\}$ and $\left\{\omega_{i} \mid i=1,2, \cdots, N\right\}$ belong to a lattice where join $U$ 
and meet $\cap$ operations are defined by which to deduce the generalised notion of maximum and minimum of any finite sets of elements. The connections between two spacetime slots of the same subjective existence are defined in the following way. For the moment we shall make use of the abbreviated notion ${ }_{\alpha} L_{\omega}$ in the place of ${ }_{\alpha} \mathcal{L}(\mathscr{P})_{\omega}$.

(i) For $\omega_{1}=\alpha_{2}$ we have $\alpha_{1} L_{\omega_{2}}={ }_{\alpha} L_{\omega_{1}}{ }^{\cdot \omega_{1}} L_{\omega_{2}}$

(ii) For $\alpha_{1}<\alpha_{2}<\omega_{1}<\omega_{2}$, we have

$$
\begin{aligned}
& { }_{\alpha_{1}} L_{\omega_{1}}={ }_{\alpha_{1}} L_{\alpha_{2}} \cdot \alpha_{2} L_{\omega_{1}} \\
& \alpha_{2} L_{\omega_{2}}={ }_{\alpha_{2}} L_{\omega_{1} \cdot \omega_{1}} L_{\omega_{2}} \\
& { }_{\alpha_{1}} L_{\omega_{2}}={ }_{\alpha_{1}} L_{\alpha_{2} \cdot \alpha_{2}} L_{\omega_{1} \cdot \omega_{1}} L_{\omega_{2}} .
\end{aligned}
$$

(iii) For $\omega_{1}<\alpha_{2}$, we want a generalised interpretation of (3.13) by introducing a virtual space time slot ${ }_{\omega_{1}} L_{\alpha_{2}}$ associated with the subjective existence $\mathscr{P}$ by which we have

$$
{ }_{\alpha_{1}} L_{\omega_{1}} \cdot \alpha_{2} L_{\omega_{2}}={ }_{\alpha_{1}} L_{\omega_{1}} \cdot \omega_{1} L_{\alpha_{2}} \cdot \alpha_{2} L_{\omega_{2}} .
$$

It is noted that $\omega_{\omega_{1}} L_{\alpha_{2}}$ is so far admitted to be introduced under the condition that ${ }_{\alpha_{1}} L_{\omega_{1}}$ and $\alpha_{2} L_{\omega_{2}}$ have their respective substantial verification.

(iv) For $\alpha_{1}<\alpha_{2}<\omega_{2}<\omega_{1}$, we have

$$
{ }_{\alpha_{1}} L_{\omega_{1}}={ }_{\alpha_{1}} L_{\alpha_{2}} \cdot \alpha_{2} L_{\omega_{2}} \cdot \omega_{2} L_{\omega_{1}}
$$

(v) In general, the combination of (i), (ii), (iii) and (iv) leads us to the connected space-time slots associated with the same subjective existence

$$
{ }_{\alpha} L_{\beta} \cdot{ }_{\gamma} L_{\delta}={ }_{\alpha \cap \gamma} L_{\beta \cup \tilde{o}}
$$

and hence to

$$
{ }_{\alpha_{1}} L_{\omega} \cdot \alpha_{2} L_{\omega_{2}} \cdots{ }_{\alpha_{N}} L_{\omega_{N}}={ }_{\alpha_{1} \cap \alpha_{2} \cap \cdots \cap \alpha_{N}} L_{\omega_{1} \cup \omega_{2} \cup \cdots \cup \omega_{N}} .
$$

After these considerations we are now proposing to introduce a sequence of time slots associated with each local space of subjective existence $\mathcal{L}(\mathscr{P}),\left\{\alpha_{i} \mathcal{L}(\mathscr{P}) \omega_{i} \mid i=1,2, \cdots, N\right\}$ which we may call local time-space or local space-temporal existence.

\subsection{Informatical aspects of local space-temporal existence}

The topics will be discussed from the standpoint of KIPS component analysis. It is noted that the local time-space of a subjective existence can be recognised to derive from the following three informatical functions associated with the KIPS components:

(i) A confrontation of subjective existence $\mathscr{P}$ with his (or her) view $V$ with the outer surrounding world $\mathscr{W}$, that is, environment, induces a formation of local space $\mathcal{L}(\mathscr{P})$.

(ii) The scheme of information materials in flow $\mathscr{F}_{D}$ associated with the KIPS of the subjective existence $\mathscr{P}$ with the view $V$ induces a time duration of the local space $\mathcal{L}(\mathscr{P})$ so far as its activity should be maintained, and hence, in combination of (i), the local space-temporal existence ${ }_{\alpha} \mathcal{L}(\mathscr{P})_{\omega}$.

(iii) Other schemes of time duration associated with the local space $\mathcal{L}(\mathscr{P})$ comes. from the brainware of the first and that of the second kind, $B R W(1)$ and $B R W(2)$, whose storages do certainly require their rerpective duration of time. 


\section{Generalised Relational Ecosphere Formation of Subjective Informatical Existences through Space-Time Construction}

Let us assume an assemble of $N$ subjective existences $\left\{\mathscr{P}_{k}\right\}(k=1,2, \cdots, N)$ to each of which there is associated with their respective KIPS having its nine components in general. It is the purpose of the present Section to formulate a generalised relational ecosphere securing both their coexistence and their mutal communicative dependencies by establishing some universal time-space constituion. We shall start with the assumption that each subjective existence $\mathscr{P}_{k}$ has its own sequence of local space temporal slots representations $\left\{a_{k_{i}} \mathcal{L}\left(\mathscr{Q}_{k}\right)_{\omega_{k_{i}}} \mid i=1,2, \cdots, T_{k}\right\} \quad(k=1,2, \cdots, N)$.

Our general formulation methodology is to appeal to what we call the principle of socialization being based upon connectivity relations among a set of sequences of local space-temporal represensations which are associated with individual subjective existences. Our methodology will be implemented by the use of generalised category, namely a category with paired morphoids which is defined in the following way.

$\left(1^{\circ}\right)$ Mathemtical formation of category with paired morphids

DEFINITION 4.1. A metacategory with paired morphoids is defined by a set of paired morphisms which satisfy the following conditions C.I.-C.IV.

C.I. To each paired morphoid $\alpha\left(\begin{array}{l}f \\ p\end{array}\right)_{\beta}$ there exist a pair of two objects $A$ and $B$ where $A$ and $B$ are called the domain of $\alpha\left(\begin{array}{l}f \\ p\end{array}\right)_{\beta}$ and the codomain of ${ }_{\alpha}\left(\begin{array}{l}f \\ p\end{array}\right)_{\beta}$ respectively and denoted by

(i) $A=\operatorname{dom}{ }_{\alpha}\left(\begin{array}{l}f \\ p\end{array}\right)_{\beta}, \quad B=\operatorname{cod}{ }_{\alpha}\left(\begin{array}{l}f \\ p\end{array}\right)_{\beta}$

(ii) ${ }_{\alpha}\left(\begin{array}{l}f \\ p\end{array}\right)_{\beta}: A \longrightarrow B$.

C. II. Under the conditions that

$$
\operatorname{dom}{ }_{\beta}\left(\begin{array}{l}
g \\
q
\end{array}\right)_{\gamma}=\operatorname{cod}{ }_{\alpha}\left(\begin{array}{l}
f \\
p
\end{array}\right)_{\beta},
$$

where

$$
\alpha\left(\begin{array}{l}
f \\
p
\end{array}\right)_{\beta}: A \longrightarrow B, \quad \beta\left(\begin{array}{l}
g \\
q
\end{array}\right)_{\gamma}: B \longrightarrow C,
$$

we have

$$
{ }_{\alpha}\left(\begin{array}{c}
f \\
p
\end{array}\right)_{\beta}{ }_{\beta}\left(\begin{array}{l}
g \\
q
\end{array}\right)_{\gamma}={ }_{\alpha}\left(\begin{array}{c}
f \circ q \\
p * q
\end{array}\right)_{\gamma} .
$$

Consequently we can and we shall write

$$
\operatorname{dom}\left({ }_{\alpha}\left(\begin{array}{c}
f \circ g \\
p * q
\end{array}\right)_{\gamma}\right)=A, \quad \operatorname{cod}\left({ }_{\alpha}\left(\begin{array}{c}
f \circ g \\
p * q
\end{array}\right)_{\gamma}\right)=C .
$$

C.III. If we have three paired morphoids

$$
\alpha\left(\begin{array}{l}
f \\
p
\end{array}\right)_{\beta}: A \longrightarrow B, \quad \beta\left(\begin{array}{l}
g \\
q
\end{array}\right)_{\gamma}: B \longrightarrow C, \quad{ }_{\gamma}\left(\begin{array}{l}
h \\
r
\end{array}\right)_{\delta}: C \longrightarrow D,
$$

then we have 


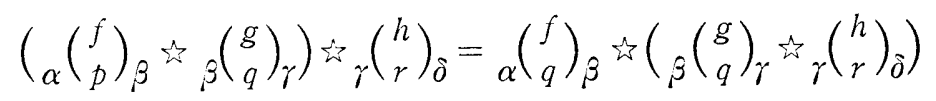

leading to

$$
\alpha\left(\begin{array}{c}
(f \circ g) \circ h \\
(p * q) * r
\end{array}\right)_{\delta}={ }_{\alpha}\left(\begin{array}{c}
f \circ(g \circ h) \\
p *(q * r)
\end{array}\right)_{\delta}
$$

C.IV. For any paired morphoid $\alpha_{\alpha}\left(\begin{array}{l}f \\ p\end{array}\right)_{\beta}$ such that we have

$$
\alpha\left(\begin{array}{l}
f \\
q
\end{array}\right)_{\beta}: A \longrightarrow B
$$

there exist two paired morphoids $\beta_{\beta}\left(\begin{array}{l}1_{A} \\ u_{A}\end{array}\right)_{\beta}$ and ${ }_{\alpha}\left(\begin{array}{c}1_{B} \\ u_{B}\end{array}\right)_{\alpha}$ such that

$$
\left\{\begin{array}{lll}
\text { (i) } & f \circ 1_{A}=f, & p \circ u_{A}=p \\
\text { (ii) } & 1_{B} \circ f=f, & u_{B} \circ p=p,
\end{array}\right.
$$

giving

$$
\begin{aligned}
& \left(\begin{array}{l}
f \\
p
\end{array}\right)_{\beta} \text {; }{ }_{\beta}\left(\begin{array}{l}
1_{A} \\
u_{A}
\end{array}\right)_{\beta}={ }_{\alpha}\left(\begin{array}{l}
f \circ 1_{A} \\
p * u_{A}
\end{array}\right)_{\beta}={ }_{\alpha}\left(\begin{array}{l}
f \\
p
\end{array}\right)_{\beta} \\
& \alpha\left(\begin{array}{l}
1_{B} \\
u_{B}
\end{array}\right)_{\alpha} \text { i }{ }_{\alpha}\left(\begin{array}{l}
f \\
p
\end{array}\right)_{\beta}={ }_{\alpha}\left(\begin{array}{l}
1_{B} \circ f \\
u_{B} * p
\end{array}\right)_{\beta}={ }_{\alpha}\left(\begin{array}{l}
f \\
p
\end{array}\right)_{\beta} .
\end{aligned}
$$

DEFINITION 4.2. A metacategory with paired morphoids is called a category with paired morphoids, if it satisfies the following conditions $C^{*} I-C^{*} V$.

$C^{*} I$. There exists a set of objects denoted by $\Omega=O b(C)$.

$C^{*} I V$. For any pair of objects $A$ and $B$ belonging to $O b(C)$, there exists a set of paired morphoids denoted by $\operatorname{Hom}(A, B)$.

$C^{*} I I I$. For any two pairs of objects $(A, B)$ and $(C, D)$ such that $(A, B) \neq(C, D)$, that is, at lest one of two identities $A=C$ and $B=D$ does not hold true, then $\operatorname{Hom}(A, B)$ $\cap \operatorname{Hom}(C, D)=\phi$.

$C^{*} I V$. For each object $A$ belonging to $\Omega,{ }_{\beta}\left(\begin{array}{l}1_{A} \\ u_{A}\end{array}\right)_{\beta}$ belongs to $\operatorname{Hom}(A, A)$ provided that there exists at least one paired morphoid of the type ${ }_{\alpha}\left(\begin{array}{l}f \\ p\end{array}\right)_{\beta}$ belonging to $H(A, A)$.

$C^{*} V$. For any triple of objects $A, B$ and $C$ belonging to $O b(C), H^{(2)}(A, C ; B)$ is defined as the product

$$
H^{(1)}(A, B) \times H^{(1)}(B, C),
$$

which satisfies the following conditions (i )-(iii).

(i) $\operatorname{Hom}^{(1)}(A, B) \triangleq \operatorname{Hom}(A, B)$

(ii) For $\forall_{\alpha}\left(\begin{array}{l}f \\ p\end{array}\right)_{\beta} \in H^{(1)}(A, B)$ and $\forall_{\beta}\left(\begin{array}{l}g \\ q\end{array}\right)_{\gamma} \in H^{(1)}(B, C)$,

we have

$$
\alpha\left(\begin{array}{c}
f \circ g \\
p * q
\end{array}\right)_{\gamma} \in H^{(2)}(A, C ; B)
$$


(iii) For $\forall{ }_{\alpha}\left(\begin{array}{l}h \\ r\end{array}\right)_{\gamma} \in H^{(2)}(A, C$; B) there exists a pair of two paired morphoids $\alpha\left(\begin{array}{c}f \\ p\end{array}\right)_{\beta}$ and $\beta\left(\begin{array}{l}g \\ q\end{array}\right)_{\gamma}$ such that

(a) ${ }_{\alpha}\left(\begin{array}{l}f \\ p\end{array}\right)_{\beta} \in H^{(1)}(A, B)$

(b) $\beta\left(\begin{array}{l}g \\ q\end{array}\right)_{\gamma} \in H^{(1)}(B, C)$

(c) $h=f \circ g, \quad r=p \circ q$.

The use of paired morphoids in our mathematical formulation comes from the following considerations.

A paired morphoid $\alpha\left(\begin{array}{l}f \\ p\end{array}\right)_{\beta}$ consists of three components where

(a) $f$ is an object morphism

(b) $p$ is an evaluation morphism

(c) The couple $(\alpha, \beta)$ corresponds to the couple $(\alpha, \beta)$,

which indicate an space-time slot associated with ${ }_{\alpha} \mathcal{L}(\mathscr{Q})_{\beta}$. The elements $\alpha$ and $\beta$ are called to be forehand and backhand respectively in corresponce with the beginning and the ending of an time-space slot ${ }_{\alpha} \mathcal{L}(\mathscr{P})_{\beta}$.

$\left(2^{\circ}\right)$ Generalised relational ecosphere approach to socialization of individual subjective existences having their respective local space-temporal representations The uses of category with paired morphoids will lead us to a generalised relational ecosphere approach to socialization of individual subjective existences having their respective local time-space slots. In fact there are two crucial concepts in this connection which will be introduced in the following two definitions:

DEFinition 4.3. A sequence of the $n$-th order paired morphoids for $n \geqq 3$ is defined in the following way:

$$
\begin{aligned}
& \text { (i) } H^{(n)}(A, B) \triangleq \bigcup_{c \in \Omega} H^{(n)}(A, B ; C) \\
& \text { (ii) } H^{(n)}(A, B ; C) \triangleq H^{(n-1)}(A, C) \times H^{(1)}(C, B) .
\end{aligned}
$$

Definition 4.4. A category with paired morphoids is said to be finite if it satisfies the following two conditions ( i )-(ii):

(i) $\Omega=O b(C)$ is a finite set.

(ii) The set $\mathscr{H}(A, B) \triangleq\left\{H^{(n)}(A, B) ; n=1,2,3, \cdots\right\}$ satisfies the condition of increasing chain.

For a finite category with paired morphoids we can introduce matrices of the following three kinds:

(i) Reachability matrix, which is defined as the set of $H^{(\infty)}\left(A, B=\lim _{n \rightarrow \infty} H^{(n)}(A, B)\right.$, where $A$ and $B$ run through the set $Q=O b(C)$.

(ii) Condensation matrix

(iii) Skeleton matrix. 
as we have already shown in our papers [7], [8], as a generalisation of interpretive structural modeling (ISM) due to Warfield [14], [15]. These three matrices give us a deep structural feature underlying.

It is adequate here to explain the reasons why and the procedures how our formulation appealing to category with paired morphoids contributes to establish universe timespace being valid among an ecosphere of time-space existences.

(i) To each object $U$ belonging to $\Omega=O b(C)$ there corresponds an individual subjective existence $\mathcal{Q}_{U}$.

(ii) To each object $U$ belonging to $\Omega=O B(C), H(U, U)\left(=H^{(1)}(U, U)\right.$ ) can be considered as the initially assigned set of local time-space representations associated with the subjective existence $\mathscr{Q}_{U}$.

(iii) To each pair of different objects $U$ and $V$ belonging to $\Omega=O b(C), H(U, V)$ $\left(=H^{(1)}(U, V)\right)$ can be considered as the initially assigined local time-space commuficative relation representations from $\mathscr{P}_{U}$ to $\mathscr{P}_{V}$.

(iv) The formation of the increasing sequence $\left\{H^{(n)}(U, V) \mid U, V \in \Omega=O b(C), n=1\right.$, $2, \cdots\}$ can be considered as an echo enlarging process of mutually reflections of the relations given in (ii) and (iii).

(v) The formation of the reachability matrix $\left\{H^{(\infty)}(U, V) \mid U, V \in \Omega=O b(C)\right\}$ gives the final results of the echo enlarging process, from which structural features of the mutal relationship among the set $\left\{\mathscr{P}_{U} \mid U \in \Omega=O b(C)\right\}$ can be observed.

(vi) It is noted that, to each assigned subjective existence $\mathscr{Q}_{U}$, the local time-space representation $\mathcal{L}\left(\mathscr{P}_{U}\right)$ is provided by $H^{(\infty)}(U, V)$, which is certainly generated from $H^{(1)}(U, V)$ through the echo formation process of (iv).

$\left(3^{\circ}\right)$ Generalised ecosphere approach to the assemble of $N$ KIPS's The formulations being given in the two previous paragraphes [1] and [2] of this Section can be explained in more definite form in connection with the assemble of $\mathrm{N}$ KIPS' each of which is associated with the local space-time existence $\left\{\alpha_{u} \mathcal{L}\left(\mathscr{P}_{u}\right) \beta_{u}\right\} \quad(u=1,2, \cdots, N)$. The explicit description of nine components in each KIPS should lead us to

$$
H\left(\mathcal{L}_{u}, \mathcal{L}_{v}\right)=\left\{\alpha\left(\begin{array}{l}
f \\
p
\end{array}\right)_{\beta} \mid \alpha\left(\begin{array}{l}
f \\
p
\end{array}\right)_{\beta}: \mathcal{L}_{u} \rightarrow \mathcal{L}_{v}\right\},
$$

where $f$ and $p$ are now denoted by

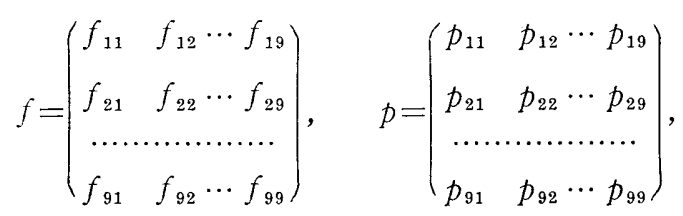

with paired morphoids $\alpha_{i j}\left(\begin{array}{c}f_{i j} \\ p_{i j}\end{array}\right)_{\omega_{i j}}$ having object morphism $f_{i j}$ and evaluation morphism $p_{i j}$

$$
\alpha_{i j}\left(\begin{array}{c}
f_{i j} \\
p_{i j}
\end{array}\right)_{\omega_{i j}}: C_{i}^{u} \longrightarrow C_{j}^{v}
$$

with $C_{i}^{u}=\operatorname{dom}\left(\begin{array}{l}f_{i j} \\ p_{i j}\end{array}\right), C_{j}^{v}=\operatorname{cod}\left(\begin{array}{l}f_{i j} \\ p_{i j}\end{array}\right)$. 


\section{Cosmos Foormations in an Ecosphere of Local Space-Temporal Existences}

The purpose of this Section is to give a few methodologies by each of which we can give a cosmos formation in an ecosphere of local space-temporal existences. Each of these methodologies comes from our own research subjects. In fact $\left(1^{\circ}\right)$ comes from system formation given in our paper [2], $\left(2^{\circ}\right)$ and $\left(3^{\circ}\right)$ from cell space approaches given in our paper [16], $\left(4^{\circ}\right)$ from our paper [19], and finally $\left(5^{\circ}\right)$ from our paper [22], where $\left(4^{\circ}\right)$ and $\left(5^{\circ}\right)$ are connected with formal neural dynamics approach developed by the present author in [22].

$\left(1^{\circ}\right)$ Lattice structure Each space-temporal slot ${ }_{\alpha} \mathcal{L}_{\omega}$ has a forehand $\alpha$ and a backhand $\omega$, where $\alpha$ and $\omega$ are elements of a lattice. This basic feature of space-temporal slot admits us to introduce the two principles of structure formation (a) sequential arrangement and (b) parallel arrangement into an ecosphere of local time-spaces, each associated with their respective individual subjective existence. In picking up some sublattices it will become possible to apply the third principle (c) black box formation within our ecosphere. An introduction of lattice structure can yield quite realistic pictures of informatical features of our ecosphere so far as we ristrict ourselves within some subecosphere, because we can be free from an assumption about existence of the absolute maximum 1 and the absolute minimum 0 for our whole system. The fourth principle (d) feedback connection can be understood to be valid within some Asian philosophical thought communities in which the time flow can be compared with circular motion in stead of straight line.

$\left(2^{\circ}\right)$ Time phase in cell space formulation Under a certain specific convention we can introduce a system of time phases in cell space formulation to a generalised relational ecosphere of individual subjective existences having their respective space-temporal slots. A specification of space configuration comes from an assumption that each individual subjective existence is located in a cell whose spatial assemble forms an $m \times n$ rectangular array. The crucial assumption is that we can introduce a notion of basic cell space which consistes of finite neighobouring cells and which can work without any interference from other cells not belonhing to the basic cell space. Under the situation a system of subdivisions of the whole cell space represented by $m \times n$ rectangle into a mutually distinct set of basic cell spaces may be assumed under some specific situation to be capable to induce a set of mutally independent time slots each of which is associated with each basic cell space. Such a system of subdivisions of the whole cell space inducing a set of mutally independent time slots is called to induce a time-phase in the whole cell space. We admit an existence of several systems of the subdivisions of the whole cell space each inducing its respective time-phase.

A transition rule among these time-phases can be defined according to a controlling rule governing the whole cell space. In fact such a controlling rule being valid among the set of basic cell space time slots induces a time cosmos among the ecosphere of individual subjective informatical existences.

Let us denote the set of all time-phases by $\boldsymbol{P}=\left\{P_{k} \mid k=1,2, \cdots, M\right\}$ associated with an ecosphere of individual subjective existences. Then a controlling rule of the timephases implies and is implied by an assignment of a sequence $\boldsymbol{K}=\left\{k_{1}, k_{2}, \cdots, k_{n}, \cdots\right\}$ 
where $1 \leqq k_{i} \leqq M,(i=1,2,3, \cdots, n, \cdots)$ inducing the sequence of time-phases $\boldsymbol{K} \boldsymbol{P}=\left(P_{k_{1}}\right.$, $\left.P_{k_{2}}, \cdots, P_{k_{n}}, \cdots\right)$.

EXAMIPLE 5.1. Random assigment Each $k_{i}$ in $\boldsymbol{K}$ is given by random sampling from the set $\{1,2, \cdots, M\}$.

EXAMPLE 5.2. Periodic assignment This is essentially the case when $k_{u p+v}=v$, for $v=0,1 ' 2, \cdots, M-1$ and $u=0,1,2, \cdots$.

$\left(3^{\circ}\right)$ Boundary constrains inherited in a lattice of roads within the ecosphere

A lattice of roads within the ecosphere can work (i) to provide with a set of boundar!es which separate each basic cell space from any other basic cell space and (ii) to impose a set of boundary constraints under which each basic cell space should work. Such a lattice of roads corresponds to a subdivision system of the whole ecosphere into a set of mutually distinct basic cell spaces and hence possibly to a time-phase. A transition of one time-phase into another natuarally involves a transition of one lattice road to another one.

The illustrations of examples and some general observations upon them, which were given in our previous paper [32] Section 4 structural aspects (pp. 42-73) and in our previous papers [33] and [34], are expected to provide us some cosmos formation in the ecosphere of local space-temporal existences.

$\left(4^{\circ}\right)$ A mathematical model of social network system In connection with his studies on neural dynamics, the present author introduces in his paper [19] a mathematical model of social information network system consisting of a set $X$ of $N$ nodes, $X=$ $\left\{X_{j} ; j=1,2, \cdots, N\right\}$ and a set of chords $\Sigma=\left\{\sigma_{j k} ; j, k=1,2, \cdots, N\right\}$ where a chord $\sigma_{j k}$ is assigned between two nodes $j$ and $k$. We assume that to each node $X_{i}$ there exist three types of function of $t$ :

(a) State function of the node $X_{i}$ at time $t$ which is denoted by $X_{i}(t)(i=1,2, \cdots, N)$.

(b) Output function from the node $X_{i}$ to the node $X_{j}$ at time $t$ which is denoted by $O_{i j}(t)(i, j=1,2, \cdots, N)$.

(c) Input function to the node $X_{i}$ from the node $X_{k}$ at time $t$ which is denoted by $e_{k i}(t)(i=1,2, \cdots, N)$. The input function to the node $X_{i}$ at time $t$ is defined by the sum

$$
e_{i}(t)=\sum_{k=1}^{N} e_{k i}(t) \text {. }
$$

(d) Memory storage function of the node $X_{i}$ at time $t$ which is denoted by $s_{i}(t)$.

Let us give a system of equations among these four types of functions :

I . State equation $\left(i=1,2, \cdots, N^{\prime}\right)$

$$
X_{i j}(t+\tau)=A_{i j}\left[X_{i j}(t), t ; e_{i}(t), s_{i}(t)\right] .
$$

II. Output equation $(i, j=1,2, \cdots, N)$

$$
O_{i j}(t+\tau)=B_{i j}\left[O_{i j}(t), t ; e_{i}(t), s_{i}(t)\right] .
$$

III. Input equation $(i, k=1,2, \cdots, N)$

$$
e_{k i}(t+\tau)=C_{k, i}\left[e_{k, i}(t), t ; s_{i}(t)\right] .
$$

IN. Memory storage equation $(i=1,2, \cdots, N)$

$$
s_{i}(t+\tau)=D_{i}\left[s_{i}(t), t ; X_{i}(t), e_{i}(t)\right] .
$$


From our standpoint appealing to its ecosphere of local time-space existences associated with each individual subjective existences, we can not assume an existence of universal time concept, but we may make apply the present formulations given in (5.01)(5.05) to each individual basic cell space consisting of several subjective existences $B_{r}=\left\{\mathscr{P}_{r, h} \mid h=1,2, \cdots, q_{r}\right\}$ with the set of the nodes $\left\{X_{h, k} \mid k=1,2, \cdots N_{k}\right\}$, provided that the following two conditions are satisfied:

(i) Automation formulation to KIPS is valid.

(ii) Mutual communications among basic cell spaces are defined in generalised relational ecoshere of basic cell spaces, and the considerations being given in Section 4, for instance, are applicable to our echsphere so as to have a universal time concept.

It is noted that morphological considerations on an inner connectivity within each individual basic cell space as well on a mutual connectivity among individual basic cell spaces will be useful in introducing (a) connectedness and separability, (b) hierachies by multistage stratifications and subnetworks and (c) hierachical structuring induced by stratified response times, as we have referred in our paper [19].

$\left(5^{\circ}\right)$ Formal neural dynamics approach This is introduced as a formal generalisation of neural dynamical equations due to Caianiello [35] and the present author [19] [22]. As we have referred in the Subsection 4.1 [4] of the paper [22], body phenomenological approaches in connection with generalized relational ecosphere provides us (i) the focusing procedure, (ii) change of central points, (iii) memory mechanism in connection with the phenomenological understanding of time concept ranging over the past, the present, and the future. It is also noted that Section 5 of the paper [22] gives our idea regarding generalised relational ecosphere approach and generalised neural dynamics.

\section{References}

[1] KITAGAWA, T.: Three coordinate system for information science appaoaches, Information Science, 5 (1973), 157-169.

[2] Kitagawa, T.: Fuzziness in informative logics, in Fuzzy sets and their applications to cognitive and decision processes, ed. by Zadeh, Fu, Tanaka and Shimura, Academic Press, (1975), 97-124.

[3] Kitagawa, T.: Mathematical approaches in theoretical cybernetics and informatics, The 8th Intern. Congress on Cybernetics, Assoc. Intern. de Cybernétique, Namur, Sept. 6-11 (1976), 267-284.

[4: Kitagawa, T.: Some methodological consideration on research automation (1) objectivization and operatorization, RIFIS, Kyushu University, RR. No. 96 (1980), 1-31.

[5] Kitagawa, T.: Datalogy and semiology in establishing knowledge-information processing systems in researches, Proceedings of the Second Intern. Conference on Hypergraphic Structured Data Base, VCU, Richmond, (1983), 1-24.

[6] KitAGAWA, T.: Generalised relative ecospheoe of knowledge-information processing systems and their implications to scientific researches, Proceedings of the Second Intern. Conference on Hypergraphic Structured Data Base, VCU, Richmond, (1983), 25-42.

[7] KITAGAwa, T.: Model and architecture of distributed data base sharing systems associated with knowledge-information processing system, Proceedings of the Second Intern. Conference on Distributed Data Base Sharing Systems, Frie Univ., Amsterdam, (1982), 295-307.

[8] KITAGAWA, T.: An informatical formutation of generatised relational ecosphere on the basis of paired categories, Intern. Conference on Cybernetics and System, (1978), 322-327.

[9] KItAgAwA, T.: A theoretical formulation of information network system and its implica- 
tion to social intelligence, IIAS-SIS, RR. No. 1 (1978), 1-21.

[10] KiTAGAwA, T.: Generalised artificial grammars and their implications to knowledge engineering approaches, IIAS-SIS, RR. 6 (1979), 1-29.

[11] KITAGAWA, T.: The logic of information sciences and its implications for control process in large systems, The second "MORMATOR" Symposium on Mathematical Methods for the Analysis of Large-Scale Systems, Prague, 1974, 15-31.

[12] Kitagawa, T.: Some contribution to the methodology of system scientific approach to environmentalogy, RIFIS, Kyushu Univ., RR. No. 71 (1976), 1-31.

[13] Kitagawa, T.: From cosmos to chaos: my scientific carreer in mathematics, statistics, and informatics, Historical Volume, Journal of Applied Probability, (1982) 165-178.

[14] WARfIELD, J.N.: Developing subsystem matrices in structural modeling, IEEE Trans. Syst., Math. Cybern., SM-4 (1974), 74-80.

[15] Warfield, J.N.: Developing interconnection mataices in structural modeling, IEEE Trans. Syst., Math. Cybern., SM-4 (1974), 81-87.

[16] Kitagawa, T. Cell space approaches in biomathematics, Math. Biosciences, 19 (1974), 27-71.

[17] [Kitagawa, T.: Dynamical systems and operators associated with a single neuronic equations, Math. Biosciences, 18 (1973), 191-244.

[18] KITAgawa, T.: Dynamical behaviours associated with a system of $N$ neuronic equations with time lag, RIFIS, (I) RR. No. 46 (1974), 1-9; (II) RR. No. 49 (1975), 1-87; (III) RR. No. 54 (1975), 1-97.

[19] Kitagawa, T.: A mathematical formulation of information network system and its implications to artificial intelligence, Modern Trends in Cybernetics and Systems, Proc. Third Intern. Congress of Cybernetics and Systems, Bucharest, 1975, IV (1977), 589-604.

[20] Kitagawa, T.: Dynamics of reverberation cycles and its implications to linguistics, RIFIS, RR. No. 56 (1975), 1-58.

[21] Kitagawa, T.: Neutral equation approach to recognition processes on the base of reveaberation and virtual dynamics, Intern. Symp. on Math. Topics in Biology, Kyoto, Sept. 11-12, (1978), 121-130.

[22] Kitagawa, T.: Formal neural dynamics, Fifth Progress in Cybernetics and Systems Research, Vol. X (1982), 161-172.

[23] Husserl, E.: Die Krisis der europäischen Wissenschaften und transzendentale Phänomeno. logie, Eine Einleitung in der phänomenologische poilosophie, Husserliana, Bd. VI, Hrsg. W. Biemal 2, Aufl. (1982).

[24] Merleau-Ponty, M.: La structure de component, Press Univ. de Frans, Paris, (1942).

[25] Merleau-Ponty, M.: Phénoménologie de la perception, Ed. Gallimard, Paris, (1945).

[26] IchikawA, H. : Body as mind (in Japanese), Keisô Shobô, (1975).

[27] KitAGawa, T.: Historical views of civilizations-prolegomena to information-oriented views of history (in Japanese), Social Information Science Series, 17, 1, Gakken (1974), 110-168.

[28] ToyenbeE, A.: A study of history, I-XII, (1934-61).

[29] Griffin, D.R.: The question of animal awareness, The Rockefeller Unipersity Press, (1976).

[30] IKEGAMI, K.: the semological structure of the English verbs of motion-a stratificational approach, Sanseido, Tokyo, (1970).

[31] TAKAsaki, J. and Umehara, T.: Learning of ancient Buddhism, Do Gen (in Japanese), Series Buddhism Thoughts, Kadokawa Shoten, 11 (1969).

[32] Kitagawa, T.: Prolegomena to cell space approaches-Information Science Approach to Biomathematics, IV, Mem. Fac. Sci., Kyushu Univ., Ser. A, XXIV, 1, (1973), 1-73.

[33] Kitagawa, T.: Oscillatory phenomena in the cell space with inhibition states $\phi$-Information Science Approach to Biomathematics, X, Bull. Math. Statist., 15, 1-2 (1972), 1-9.

34] Kitagawa, T.: An $m \times n$ cell space surrounded by the invariant boundaries and tow inhibi. tion states $\phi$-Information Science Approach to Biomathematics, XII, Bull. Math. Statist., $15,1-2$ (1972), 67-84.

[35] Cainniello, E.R.: Outline of a thought-processes and thinking machines, Journ. Theret. B!ology, 2 (1961), 204-235.

Received August 25, 1983 